\title{
Subarray-Synthesized Low-Side-Lobe Sum and Difference Patterns with Partial Common Weights
}

\author{
Ta-Sung Lee, Member, IEEE, and Te-Kung Tseng
}

\begin{abstract}
New methods of synthesizing low-side-lobe sum and difference patterns for linear arrays are described. By subarray beam forming, the sum and difference patterns exhibit a common factor, leading to a simple monopulse estimator. A procedure is proposed for the synthesis of the subarray-based sum and difference patterns which approximate a set of reference patterns in least-squares sense. Supervised nulling is incorporated as a means of suppression of strong interference. To further reduce system complexity, we impose the constraint that parts of the beam-forming weights are shared by the sum and difference channels. The relationship between the maximum number of common weights allowable and the number of subarrays used is discussed. Numerical results are presented to demonstrate the efficacy of the proposed methods.
\end{abstract}

\section{INTRODUCTION}

$\mathbf{T}$ HE design of sum and difference beam formers for amplitude-comparison monopulse arrays has received considerable attention [1]-[7]. Much work has been focused on the synthesis of patterns with side-lobe cancellation capability. Interference cancellation can be accomplished either by using uniformly low side lobes or by placing a deep null in the direction of each interferer. The latter approach requires a knowledge of the interfering directions or some kind of adaptive processing on the array data. The former approach, on the other hand, does not require any prior knowledge about the noise field and is thus simpler to implement. The price paid for low-side-lobe implementation is that the sensitivity and performance of the monopulse estimator degrade because of a wider effective main lobe.

Conventional monopulse target localization requires a knowledge of the sum and difference patterns. Typically, a look-up table is set up beforehand, from which a target bearing can be read out corresponding to a set of sum and difference data [8], [9]. With the advent of modern phased array techniques, monopulse estimators can be implemented more flexibly in that the sum and difference patterns are synthesized with two sets of properly chosen complex weights [2]-[5]. A major drawback of employing a table look-up scheme in a monopulse array is that when the beam former is operated to perform adaptive nulling against strong interferers,

Manuscript received July 24, 1992; revised December 17, 1992. This work was supported by the National Science Council of R.O.C. under grant NSC 80-E-SP-009-01A.

T.-S. Lee is with the Department of Communication Engineering, National Chiao Tung University, Hsinchu, Taiwan, Republic of China.

T.-K. Tseng was with the Department of Communication Engineering, National Chiao Tung University, Hsinchu, Taiwan. He is now with the TECOM Corporation, Hsinchu, Taiwan, Republic of China.

IEEE Log Number 9210818 the resulting sum and difference patterns vary with time such that the table must be updated every several acquisitions. As an alternative, monopulse estimators may be implemented according to some statistical parameter estimation techniques, such as maximum likelihood (ML) [10], [11]. In these methods, the target bearing is determined via a search for the maximum of the corresponding likelihood function. Although the ML approach yields statistically efficient bearing estimates, it is usually not practical owing to the high computational complexity.

In this paper, we present a new method of monopulse localization using a linear, equally-spaced (LES) array. The method is derived based on the relationship between the difference-to-sum ratio (DSR) and the target bearing under no noise/error condition. By invoking the equivalence between array patterns and polynomials for an LES array, we can convert the problem of bearing estimation into one of polynomial rooting. To alleviate the high numerical complexity incurred with large order polynomial rooting, we propose a subarray beam-forming scheme which can reduce the polynomial into a small order one. The simplified scheme exploits the fact that with subarray beam forming the sum and difference patterns share a common factor, corresponding to a common polynomial factor which can be factored out. In order to retain good estimation performance, the sum and difference patterns are so synthesized as to best approximate a set of reference patterns in least-squares (LS) sense. For the case considered herein, we choose the Chebyshev [6] and the Bayliss [7] patterns as the reference for the sum and difference channels, respectively. In the case where strong directional jammers exisit, it is necessary to perform adaptive nulling to avoid track breaking. Invoking the concept of simultaneous nulling [2], [3], we impose the constraint that a null be placed in each of the jamming directions in both the sum and difference patterns. Since these nulls are common to both patterns, they can be associated with the aforementioned common pattern factor.

To further reduce system complexity, we incorporate the concept of partial common weights; i.e., some of the weights associated with the difference beams are directly copied from the weights associated with the sum beam, with the sign reversed for each half. It is shown that under subarray processing, the number of common weights allowable is bounded above by approximately twice the number of subarrays used. For practical sum and difference excitation distributions, the appropriate number of common weights equals one half the number of the full array elements. In this case, the number of 
independent weights can be reduced by a factor of $4 / 3$, and the order of polynomial rooting in bearing estimation can be reduced by at most a factor of 4 .

\section{NOTATION AND DERIVATION OF MONOPULSE ESTIMATOR}

We first define some of the notation frequently used in this paper:

1) $\boldsymbol{M}^{T}\left(\boldsymbol{v}^{T}\right)$ : transpose of matrix $\boldsymbol{M}$ (vector $\boldsymbol{v}$ )

2) $\boldsymbol{M}^{H}\left(\boldsymbol{v}^{H}\right)$ : conjugate transpose of matrix $\boldsymbol{M}$ (vector $\boldsymbol{v}$ )

3) $\boldsymbol{I}_{n}: n \times n$ identity matrix

4) $\mathbf{1}_{n}: n \times 1$ all ones vector

5) $O_{n}:\left(O_{m \times n}\right) n \times n(m \times n)$ zero matrix

6) $\mathbf{o}_{n}: n \times 1$ zero vector

7) $\|\boldsymbol{v}\|:$ 2-norm of vector $\boldsymbol{v}$

8) $\boldsymbol{M}\left(n_{1}: n_{2},:\right)$ : submatrix consisting of the $n_{1}$ th to $n_{2}$ th row of matrix $\boldsymbol{M}$

9) $\boldsymbol{M}\left(:, n_{1}: n_{2}\right)$ : submatrix consisting of the $n_{1}$ th to $n_{2}$ th column of matrix $\boldsymbol{M}$

10) $v\left(n_{1}: n_{2}\right)$ : subvector consisting of the $n_{1}$ th to $n_{2}$ th component of vector $\boldsymbol{v}$.

We here consider the scenario of a single target illuminated by a linear array with $M$ identical elements uniformly spaced by a half wavelength. The target is assumed to be in the far field of the array such that the echoes received can be modeled as plane waves. In this case, the array data (in complex envelops) received at the $M$ elements at a certain sampling instant can be put in the following $M \times 1$ vector form:

$$
\boldsymbol{x}=\xi \boldsymbol{a}_{M}\left(u_{t}\right)+\boldsymbol{n},
$$

where $u_{t}=\sin \left(\theta_{t}\right)$, with $\theta_{t}$ representing the bearing of the target with respect to the broad side of the array as shown in Fig. 1. The complex scalar $\xi$ represents the echo received at some reference point of the array. The $M \times 1$ vector $\boldsymbol{a}_{M}\left(u_{t}\right)$ is the array direction vector evaluated at $u=u_{t}$, accounting for the phase variation across the array caused by the echoes. Finally, the $M \times 1$ vector $\boldsymbol{n}$ is composed of the complex noise envelopes present at the $M$ elements. Setting the reference point of the array to be at its geometric center, we have

$$
\begin{aligned}
\boldsymbol{a}_{M}(u)=\left[e^{-j \pi\left(\frac{M-1}{2}\right) u}, e^{-j \pi\left(\frac{M-3}{2}\right) u}\right. \\
\\
\left.\quad \cdots, e^{j \pi\left(\frac{M-3}{2}\right) u}, e^{j \pi\left(\frac{M-1}{2}\right) u}\right]^{T} .
\end{aligned}
$$

We observe that the array direction vector exhibits the conjugate symmetric (CS) and Vandermonde structures. Amplitude comparison operation dictates that the appropriate sum and difference beams be formed in the vicinity of the target bearing. Mathematically speaking, the beam-forming operation converts $\boldsymbol{x}$ into the sum and difference data:

$$
\begin{gathered}
\Sigma=\boldsymbol{s}^{H} \boldsymbol{x}=\xi \boldsymbol{s}^{H} \boldsymbol{a}_{M}\left(u_{t}\right)+\boldsymbol{s}^{H} \boldsymbol{n} \\
\Delta=\boldsymbol{d}^{H} \boldsymbol{x}=\xi \boldsymbol{d}^{H} \boldsymbol{a}_{M}\left(u_{t}\right)+\boldsymbol{d}^{H} \boldsymbol{n},
\end{gathered}
$$

where $s$ and $\boldsymbol{d}$ denote the $M \times 1$ sum and difference beamforming weight vectors, respectively.

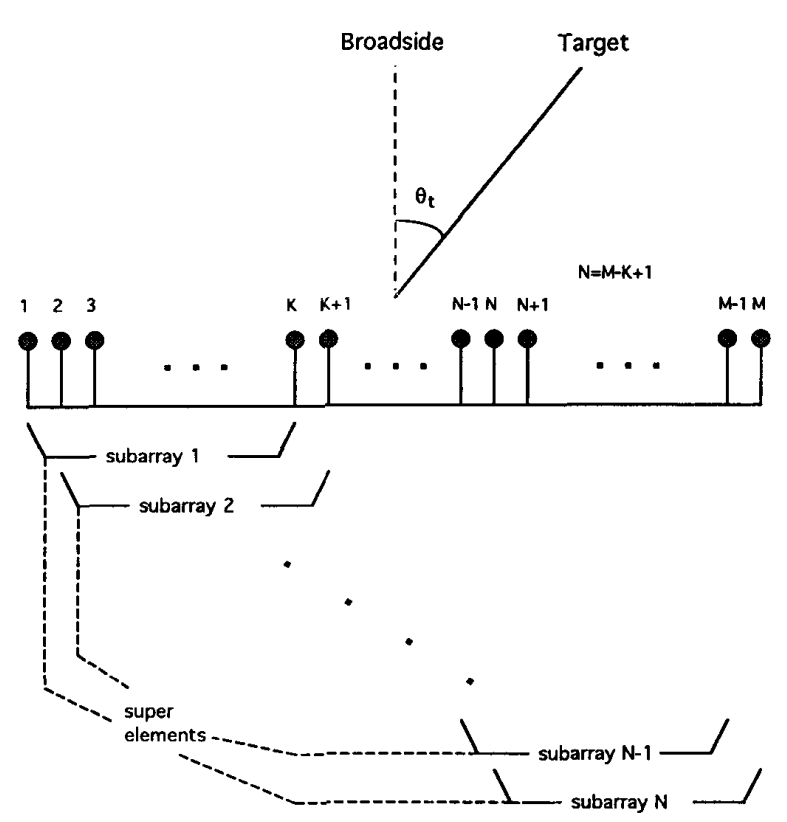

Fig. 1. Array geometry.

\section{A. Bearing Estimation with Subarray-Formed Sum and Difference Beams}

In conventional monopulse estimation, the target bearing estimate $\hat{u}_{t}$ is determined by equating the DSR and the ratio of the difference pattern to the sum pattern:

$\rho=\frac{\Delta}{\Sigma}=\left.\left.\frac{\boldsymbol{d}^{H} \boldsymbol{a}_{M}(u)}{\boldsymbol{s}^{H} \boldsymbol{a}_{M}(u)}\right|_{u=\hat{u}_{t}} \Longrightarrow(\boldsymbol{d}-\rho \boldsymbol{s})^{H} \boldsymbol{a}_{M}(u)\right|_{u=\hat{u}_{t}}=0$

where $d$ - $\rho s$ represents a beam-forming weight vector producing a null in the direction of $\hat{u}_{t}$. Defining

$$
z_{M}=\left[1, z, z^{2}, \cdots, z^{M-1}\right]^{T}
$$

we may rewrite (4) as a polynomial equation of order $M-1$ :

$$
\left.(\boldsymbol{d}-\rho \boldsymbol{s})^{H} z_{M}\right|_{z=\hat{z}_{t}}=0,
$$

with $z=e^{j \pi u}$. An obvious way to obtain $\hat{u}_{t}$ is to determine $\hat{z}_{t}=\left|\hat{z}_{t}\right| e^{j \pi \hat{u}_{t}}$ as a root of (6) close to the unit circle such that $\hat{u}_{t}$ is in the vicinity of the boresight of the array. If $s$ and $\boldsymbol{d}$ do not vary over the period of estimation, then a table relating $\hat{u}_{t}$ and $\rho$ can be constructed and stored for use.

The above described scheme is inefficient in that it requires rooting an $(M-1)$ th-order polynomial in order to determine a single parameter, $\hat{u}_{t}$. This is more significant as the number of elements, $M$, increases. The table look-up method is simple, but is of limited use for arrays performing adaptive nulling such that the sum and difference weight vectors vary with time. As a remedy, we propose a subarray beam-forming scheme that leads to substantial simplification in bearing estimation. 


\section{B. Simplifications Arising from Subarray Beam Forming}

Consider grouping $K$ successive elements of an $M$-element LES array into an LES subarray of size $K$, as shown in Fig. 1. There are total of $N=M-K+1$ such subarrays, and any two adjacent subarrays have $K-1$ elements in common. Suppose that a beam former is attached to each of the subarrays with the same $K \times 1$ weight vector $c=$ $\left[c_{1}, \cdots, c_{K}\right]^{T}$. These tapered subarrays may be viewed as "superelements" with patterns determined by $c$. The sum and difference beams are then formed, with $N \times 1$ weight vectors $\boldsymbol{g}=\left[g_{1}, \cdots, g_{N}\right]^{T}$ and $\boldsymbol{h}=\left[h_{1}, \cdots, h_{N}\right]^{T}$, respectively, treating these superelements as the elements of an $N$-element LES array. Invoking the principle of phased arrays [12], the resulting sum and difference patterns can be expressed as

$$
\begin{gathered}
s(u)=\boldsymbol{s}^{H} \boldsymbol{a}_{M}(u)=g(u) c(u) \\
d(u)=\boldsymbol{d}^{H} \boldsymbol{a}_{M}(u)=h(u) c(u),
\end{gathered}
$$

where $c(u)=c^{H} a_{K}(u)$ represents the superelement pattern, and $g(u)=g^{H} a_{N}(u)$ and $h(u)=h^{H} a_{N}(u)$ represent the "array factors" associated with the sum and difference beams. $\boldsymbol{a}_{K}(u)$ and $\boldsymbol{a}_{N}(u)$ are given by (2), with $M=K$ and $M=N$, respectively. Note from (7) that the sum and difference patterns share a common factor $c(u)$. Substituting (7) into (4) and factoring $c(u)$ out yields

$$
\left.(\boldsymbol{h}-\rho \boldsymbol{g})^{\boldsymbol{H}} \boldsymbol{a}_{N}(u)\right|_{u=\hat{u}_{t}}=0 .
$$

The feasibility of using (8) in place of (4) lies in the fact that $c\left(\hat{u}_{t}\right) \neq 0$. This is usually true since $\hat{u}_{t}$ should be close to the boresight angle at which the sum beam has the maximum gain. As a practical problem, the array elements may suffer from gain/phase uncertainties owing to the impairment of electronic circuitry, errors in element locations, or mutual coupling. If the errors within a subarray are different for the sum and difference beam formers, or if different subarrays have different random errors, then the factorizations in (7) no longer hold. In this case, the superelement pattern will not cancel in the DSR, and using (8) results in an error in $\hat{u}_{t}$. A remedy would be to employ array calibration [13] to remove the gain/phase uncertainties.

Equation (8) is in fact associated with an $N$-element LES array so that it can be solved with a much lower complexity than (4) for $N \ll M$. We may similarly convert (8) into an $(N-1)$ th-order polynomial equation:

$$
\left.(\boldsymbol{h}-\rho \boldsymbol{g})^{H} z_{N}\right|_{z=\hat{z}_{t}}=0,
$$

where $z_{N}$ is given by (5) with $M=N$. In this case, we need only root an $(N-1)$ th-order polynomial to determine $\hat{u}_{t}$. As a demonstration, consider the simplest case of $K=M-1$, or $N=2$. According to the general characteristics of the sum and difference patterns, we impose that $h(u)$ has a single null at $u_{o}$, the boresight angle of the array, and $g(u)$ has a single null at $u_{o} \pm 1$. Note that $u_{o}+1$ and $u_{o}-1$ correspond to the same angle since the angle spectrum is periodic with period 2 for the case considered herein. Incorporation of these facts leads to the following expressions:

$$
\begin{aligned}
& g(u)=\alpha\left(e^{j \pi u}+e^{j \pi u_{o}}\right) \\
& h(u)=\beta\left(e^{j \pi u}-e^{j \pi u_{o}}\right),
\end{aligned}
$$

where $\alpha$ and $\beta$ are nonzero scalars. Substituting (10) into (8) and solving for $\hat{u}_{t}$ yields

$$
\hat{u}_{t}=u_{o}+\frac{1}{\pi} \arg \left\{\frac{\beta+\rho \alpha}{\beta-\rho \alpha}\right\} .
$$

Despite its simplicity, the sum and difference beams formed with $K=M-1$ exhibit undesired side-lobe behavior. To see this, we check from (10) that $g(u)$ is essentially a half cycle cosine wave with the maximum at $u_{o}$, whereas $h(u)$ is essentially a half cycle sine wave with the maximum at $u_{o} \pm 1$. We then see from (7) that the side-lobe level of the sum pattern tends to decrease as the off-boresight angle $\left|u-u_{o}\right|$ increases, whereas the opposite is true for the difference pattern. It is thus not possible for both the sum and difference patterns to exhibit uniformly low side lobes as desired. This represents a trade-off between system complexity and pattern quality.

\section{SYNTHESIS OF SUBARRAY-BASED/LS-FIT LOW-SIDE-LOBE PATTERNS}

For convenience, we will work with $u_{o}=0$. For a nonzero boresight angle, the results can be easily modified via suitable progressive phase shifting. The pattern factorizations in (7) translate into the following polynomial factorizations:

$$
\begin{aligned}
s(z) & =\boldsymbol{s}^{H} z_{M}=g(z) c(z) \\
d(z) & =d^{H} z_{M}=h(z) c(z),
\end{aligned}
$$

where $c(z)=c^{H} z_{K}, g(z)=g^{H} z_{N}$, and $h(z)=h^{H} z_{N} \cdot z_{K}$ is given by (5) with $M=K$. Invoking the equivalence between polynomial multiplication and linear convolution, (12) can in turn be converted into the following matrix forms:

$$
\begin{aligned}
& \boldsymbol{s}=\boldsymbol{C g}=\boldsymbol{G c} \\
& \boldsymbol{d}=\boldsymbol{C h}=\boldsymbol{H c}
\end{aligned}
$$

where

$$
\mathbf{C}=\left[\begin{array}{cc}
c_{1} & 0 \\
\vdots & c_{1} \\
c_{K} & \vdots \\
\bigcirc & c_{K}
\end{array}\right]
$$

is an $M \times N$ banded Toeplitz matrix and

$$
\begin{aligned}
& \mathbf{G}=\left[\begin{array}{ccc}
g_{1} & & \bigcirc \\
\vdots & & g_{1} \\
g_{N} & \vdots \\
\bigcirc & & g_{N}
\end{array}\right] \\
& \mathbf{H}=\left[\begin{array}{cc}
h_{1} & \bigcirc \\
\vdots & h_{1} \\
h_{N} & \vdots \\
\bigcirc & h_{N}
\end{array}\right]
\end{aligned}
$$

are $M \times K$ banded Toeplitz matrices. Note that in (13) we have invoked the commutativity of linear convolution. 


\section{A. Synthesis of Quiescent Patterns}

The problem of determining a set of "optimum" subarraysynthesized sum and difference patterns can be formulated as that of finding a "best" combination of $\boldsymbol{c}, \boldsymbol{g}$, and $\boldsymbol{h}$, in terms of a prescribed criterion. For example, the practical desired property of uniformly low side lobes can be achieved with the Chebyshev weighting [6] (for the sum pattern), and the Bayliss weighting [7] (for the difference pattern). A natural way to retain low side lobes with the proposed beam former is then to make $\boldsymbol{s}$ and $\boldsymbol{d}$ as close to the Chebyshev and Bayliss weight vectors, respectively, as possible. Suppose that a set of "reference" weight vectors $\boldsymbol{s}_{o}$ (Chebyshev) and $\boldsymbol{d}_{o}$ (Bayliss) are chosen. A convenient measure of the "distance" between the reference and the synthesized patterns is the $L_{2}$ norm defined by

$$
\begin{aligned}
D_{2} & =\int_{-1}^{1}\left\{\left[\left(\boldsymbol{s}-\boldsymbol{s}_{o}\right)^{H} \boldsymbol{a}_{M}(u)\right]^{2}+\left[\left(\boldsymbol{d}-\boldsymbol{d}_{o}\right)^{H} \boldsymbol{a}_{M}(u)\right]^{2}\right\} d u \\
& =\left\|\boldsymbol{s}-\boldsymbol{s}_{o}\right\|^{2}+\left\|\boldsymbol{d}-\boldsymbol{d}_{o}\right\|^{2}
\end{aligned}
$$

where we have invoked Parseval's relationship for LES arrays. Our problem of interest is then to determine $\boldsymbol{c}, \boldsymbol{g}$, and $\boldsymbol{h}$ so as to minimize $D_{2}$. The problem has no closed-form solution in general. Instead of solving it with brute force, we propose that the problem be decomposed into two individual stages where in one stage we solve for the common factor $c$ and in the other we solve for the uncommon factors $g$ and $h$.

Assume that we first obtain an initial guess of $c$ (or $C$ ) and rewrite $D_{2}$ to form the following problem with the two unknowns $\boldsymbol{g}$ and $\boldsymbol{h}$ :

$$
\begin{gathered}
\min _{\boldsymbol{g}, \boldsymbol{h}}\left\|\boldsymbol{C g}-\boldsymbol{s}_{o}\right\|^{2}+\left\|\boldsymbol{C h}-\boldsymbol{d}_{o}\right\|^{2} \\
\text { subject to: } \quad \boldsymbol{h}^{H} \boldsymbol{a}_{N}(0)=\boldsymbol{h}^{H} \mathbf{1}_{N}=0
\end{gathered}
$$

where the constraint ensures that the difference pattern has a null at the boresight angle $u_{o}=0$. Equation (17) is separable for $g$ and $h$ such that the solutions are given by

$$
\begin{gathered}
\boldsymbol{g}=\left(\boldsymbol{C}^{H} \boldsymbol{C}\right)^{-1} \boldsymbol{C}^{H} \boldsymbol{s}_{o} \\
\boldsymbol{h}=\left(\boldsymbol{C}^{H} \boldsymbol{C}\right)^{-1}\left\{\boldsymbol{I}_{N}-\frac{\mathbf{1}_{N} \mathbf{1}_{N}^{T}\left(\boldsymbol{C}^{H} \boldsymbol{C}\right)^{-1}}{\mathbf{1}_{N}^{T}\left(\boldsymbol{C}^{H} \boldsymbol{C}\right)^{-1} \mathbf{1}_{N}}\right\} \boldsymbol{C}^{H} \boldsymbol{d}_{o}
\end{gathered}
$$

With the $\boldsymbol{g}$ and $\boldsymbol{h}$ (or $\boldsymbol{G}$ and $\boldsymbol{H}$ ) thus obtained, we may proceed to find the new $c$ by solving

$$
\min _{\boldsymbol{c}}\left\|\boldsymbol{G c}-\boldsymbol{s}_{o}\right\|^{2}+\left\|\boldsymbol{H} \boldsymbol{c}-\boldsymbol{d}_{o}\right\|^{2} \equiv \min _{\boldsymbol{c}}\left\|\boldsymbol{F} \boldsymbol{c}-\boldsymbol{w}_{o}\right\|^{2},
$$

where

$$
\boldsymbol{F}=\left[\begin{array}{l}
\boldsymbol{G} \\
\boldsymbol{H}
\end{array}\right]
$$

and

$$
\boldsymbol{w}_{o}=\left[\begin{array}{l}
\boldsymbol{s}_{o} \\
\boldsymbol{d}_{o}
\end{array}\right]
$$

The solution is given by

$$
\boldsymbol{c}=\left(\boldsymbol{F}^{H} \boldsymbol{F}\right)^{-1} \boldsymbol{F}^{H} \boldsymbol{w}_{o} .
$$

We may then substitute (22), along with (14), into (17) to obtain new $\boldsymbol{g}$ and $\boldsymbol{h}$. The procedure is alternately executed between (18) and (22) until the solutions converge. Simulations indicate that the algorithm converges within five to seven iterations for a suitably chosen initial guess of $c$.

It is often desirable to work with CS weight vectors, i.e.,

$$
\begin{gathered}
\tilde{I}_{M} \boldsymbol{s}=\boldsymbol{s}^{*} \\
\tilde{\boldsymbol{I}}_{M} \boldsymbol{d}=d^{*}
\end{gathered}
$$

where

$$
\tilde{\boldsymbol{I}}_{M}=\left[\begin{array}{cccc}
0 & 0 & \cdots & 1 \\
0 & \cdots & 1 & 0 \\
\vdots & . \cdot & \vdots & \vdots \\
1 & 0 & \cdots & 0
\end{array}\right]
$$

is the $M \times M$ reverse permutation matrix. There are two advantages to working with CS weight vectors. First, CS property is a necessary condition for the roots of the corresponding polynomials $s(z)$ and $d(z)$ to lie on the unit circle. This is essential since a root of $s(z)$ or $d(z)$ corresponds to a null in $s(u)$ or $d(u)$. Second, if $s$ and $d$ are CS, then $s(u)$ or $d(u)$ are real functions of $u$. This implies that $\Sigma$ and $\Delta$ have the same phase angle such that $\rho$ is a real number under no noise/error condition. The Appendix shows that if $s_{o}, d_{o}$ and the initial guess of $c$ are all CS, then the solutions for $g, h$, and $c$ in each stage of the procedure outlined in (17)-(22) are all CS as well. We then conclude that the final solutions for $s$ and $d$ constructed in accordance with (13) are both CS since the convolution of two CS vectors is another CS vector.

\section{B. Synthesis of Patterns in the Presence of Strong Interference}

If strong out-of-band active interference exists, the side lobes of the sum and difference beams may not be low enough to provide effective suppression of these undesired sources. It is then necessary to perform adaptive cancellation by putting a deep null in the direction of each of the interferers for the sum and difference beams. Since these nulls are common to $s(u)$ and $d(u)$, they should be associated with $c(u)$. Taking the supervised nulling approach [3], [14], we assume that the interfering directions are first estimated during the passive period of the radar via some kind of off-line direction-finding algorithm. The sum and difference beams are then formed accordingly to put "hard nulls" in these directions.

Let $u_{i}, i=1, \cdots, I$, be the $I$ estimated interfering directions. The execution of interference cancellation requires that

$$
\boldsymbol{s}^{H} \boldsymbol{a}_{M}\left(u_{i}\right)=d^{H} \boldsymbol{a}_{M}\left(u_{i}\right)=c^{H} a_{K}\left(u_{i}\right)=0 \quad i=1, \cdots, I .
$$

This says that $u_{i}, i=1, \cdots, I$, are $I$ "common nulls" associated with $s$ and $\boldsymbol{d}$. Incorporation of this $a$ priori knowledge in the aforementioned LS-fit procedure leads to the two-stage problem described by (17) and

$$
\begin{gathered}
\min _{\boldsymbol{c}}\left\|\boldsymbol{F} c-\boldsymbol{w}_{o}\right\|^{2} \\
\text { subject to: } \quad c^{H} A_{I}=0
\end{gathered}
$$


with

$$
\boldsymbol{A}_{I}=\left[\boldsymbol{a}_{K}\left(u_{1}\right)\left|\boldsymbol{a}_{K}\left(u_{2}\right)\right| \cdots \mid \boldsymbol{a}_{K}\left(u_{I}\right)\right] .
$$

The solution to (26) is similar in form to (18):

$$
\begin{aligned}
\boldsymbol{c}=\left(\boldsymbol{F}^{H} \boldsymbol{F}\right)^{-1}\left\{\boldsymbol{I}_{M}-\boldsymbol{A}_{I}\left[\boldsymbol{A}_{I}^{H}\left(\boldsymbol{F}^{H} \boldsymbol{F}\right)^{-1} \boldsymbol{A}_{I}\right]^{-1}\right. \\
\left.\cdot \boldsymbol{A}_{I}^{H}\left(\boldsymbol{F}^{H} \boldsymbol{F}\right)^{-1}\right\} \boldsymbol{F}^{H} \boldsymbol{s} .
\end{aligned}
$$

The procedure is alternately executed between (18) and (28) until the solutions converge. Similar to what is shown in the Appendix, we observe that the final sum and difference weight vectors are CS, provided that $\boldsymbol{s}_{o}, \boldsymbol{d}_{o}$, and the initial guess of $c$ are all $\mathrm{CS}$.

To ensure that the superelement pattern has the desired sidelobe property in the region of no jammers, it is necessary to provide a sufficient degree of freedom for $c$ in the LS-fit problem of (26). This means that the subarray size $K$ should be large enough compared with $I$. On the other hand, $K$ should be kept small in order to retain the proximity between the synthesized and reference patterns. This represents a trade-off in choosing $K$ or $N$.

\section{Numerical Examples}

Numerical simulations were conducted to demonstrate the efficacy of the proposed beam-forming techniques. The array employed was linear, consisting of $M=20$ identical elements uniformly spaced by half a wavelength. For all cases, the Chebyshev and the Bayliss patterns, both with $-30 \mathrm{~dB}$ sidelobe level, were used as the reference sum and difference patterns, respectively. The boresight angle was set to be $u_{o}=0$ as assumed.

The first set of examples investigate the sum and difference patterns synthesized in accordance with the previously developed LS-fit procedure. As a complete demonstration, Fig. 2 shows the superposition of the patterns synthesized in accordance with (17)-(22) for $N=2,4$, and 6 . It is evident that the proximity between the synthesized and the reference patterns improves as $N$ increases. The disparity between the side lobes of the sum and difference patterns for $N=2$ confirms our earlier statement that uniform side lobes cannot be achieved for both patterns in such a case. We observe that the sum and difference patterns exhibit $K-1=M-N$ "common nulls" corresponding to the common factor $c(u)$.

To examine the effect of adaptive nulling of the proposed beam-forming scheme, three hard nulls were formed at $-40^{\circ}$, $30^{\circ}$ and $50^{\circ}$, corresponding to three jamming directions, in accordance with (26) and (27). Fig. 3 shows the resulting patterns for $N=4$. We find that the three nulls are present in both the sum and difference patterns, indicating that they are associated with $c$. We also note that low sidelobes were retained by these modified patterns. This ascertains the effectiveness of the LS-fit approach.

For all of the above examples, the initial guess of $c$ was chosen as $c=1_{K}$. The algorithm was terminated if $\left\|\boldsymbol{c}^{k+1}-\boldsymbol{c}^{k}\right\|<0.01\left\|\boldsymbol{c}^{k}\right\|$, where $\boldsymbol{c}^{k}$ denotes the $\boldsymbol{c}$ vector obtained at the $k$ th iteration. We found that, on an average, the algorithm converged within seven iterations.

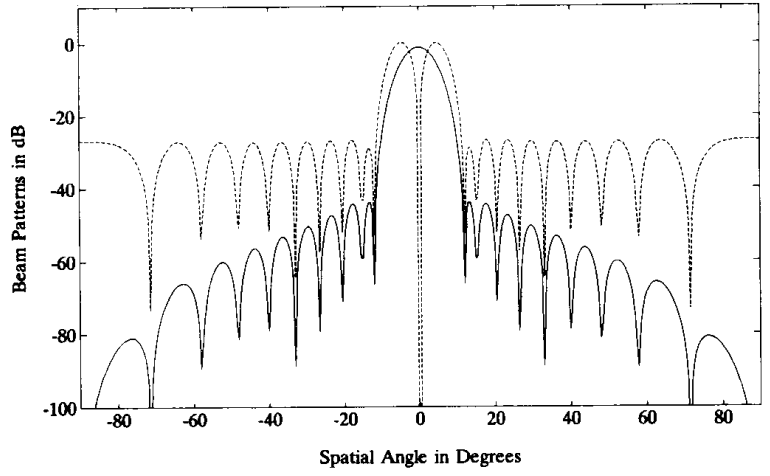

(a)

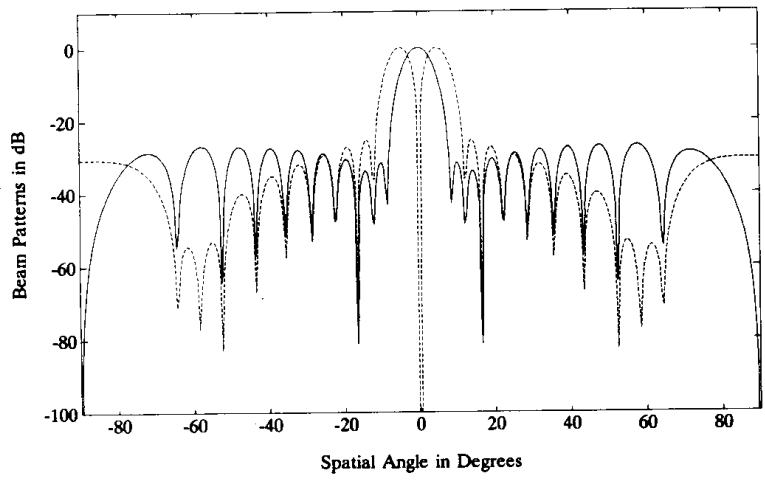

(b)

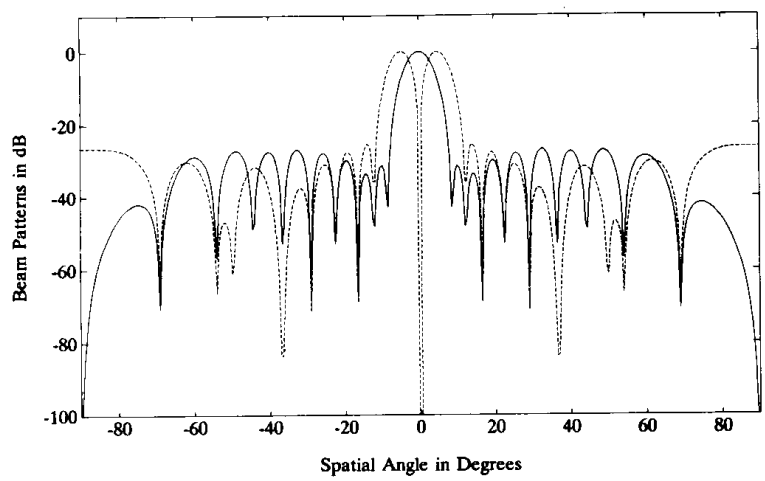

(c)

Fig. 2. Superposition of the subarray-based/LS-fit patterns synthesized with a 20-element LES array. (a) $N=2$. (b) $N=4$. (c) $N=6$. Solid line: sum. Dashed line: difference.

\section{COMMON WEIGHTS IMPLEMENTATION}

A simple way to implement the sum and difference beam formers is to determine the weights for the sum channel first, and then reverse the sign of half of the weights for the difference channel. The simplification in system complexity is due to the fact that only a single set of weights is needed. However, working with full common weights in the proposed subarray beam-forming scheme will lead to a poor approximation between the synthesized patterns and the reference patterns. 


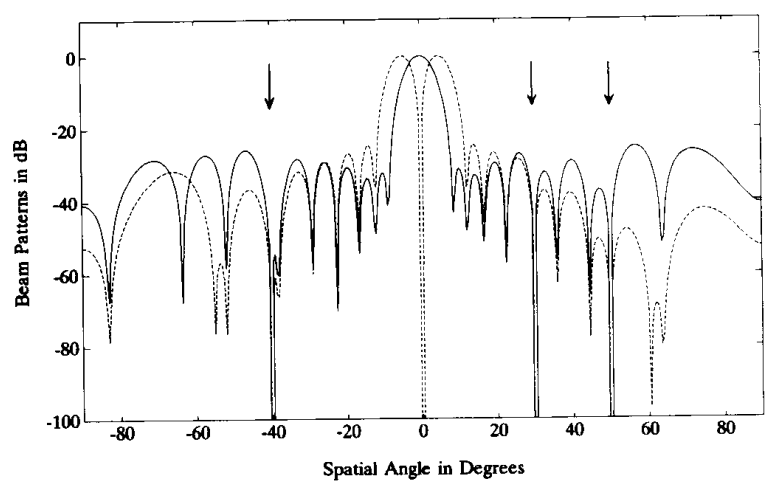

Fig. 3. Superposition of the subarray-based/LS-fit interference cancellation patterns synthesized with a 20-element LES array. $N=4$. Solid line: sum. Dashed line: difference.

This is because there are too many constraints imposed upon the beam formers. As a remedy, we propose the use of partial common weights.

\section{A. Synthesis of Patterns with Partial Common Weights}

To determine which of the $M$ weights should be the common ones, we consider first that the excitation distribution associated with the Chebyshev beam resembles a half cycle cosine wave; i.e., it is even symmetric with the maximum at the center and the minimum at both ends [6]. On the other hand, the excitation distribution associated with the Bayliss beam resembles a full cycle sine wave; i.e., it is odd symmetric with the minimum at both ends [7]. It is thus conceivable that commonality should be imposed upon the weights near both ends of the aperture. Now suppose that the first $J$ weights from either end of the aperture are common. Also, assume for convenience that $M=2 L$ is even. Construction of the sum and difference beams in this fashion and incorporating the CS structure lead to the following forms for $s$ and $d$ :

$$
\begin{gathered}
\boldsymbol{s}=\left[\begin{array}{c}
\boldsymbol{r} \\
\vdots \\
\tilde{\boldsymbol{I}}_{J} \boldsymbol{r}^{*}
\end{array}\right] \\
\boldsymbol{d}=\left[\begin{array}{c}
j \boldsymbol{r} \\
\vdots \\
-j \tilde{\boldsymbol{I}}_{J} \boldsymbol{r}^{*}
\end{array}\right]
\end{gathered}
$$

where $\boldsymbol{r}$ is a $J \times 1$ vector, and $\tilde{\boldsymbol{I}}_{J}$ is given by (24) with $M=J$. Expressions in (29) lead to the following relationship:

$$
\Gamma_{s} s=\Gamma_{d} d
$$

where

$$
\begin{aligned}
\boldsymbol{\Gamma}_{\boldsymbol{s}} & =\left[\begin{array}{cc}
j \boldsymbol{I}_{J} & \boldsymbol{O}_{J \times(M-J)} \\
\boldsymbol{O}_{J \times(M-J)} & -j \boldsymbol{I}_{J}
\end{array}\right] \\
\boldsymbol{\Gamma}_{\boldsymbol{d}} & =\left[\begin{array}{cc}
\boldsymbol{I}_{J} & \boldsymbol{O}_{J \times(M-J)} \\
\boldsymbol{O}_{J \times(M-J)} & \boldsymbol{I}_{J}
\end{array}\right]
\end{aligned}
$$

We first investigate the structures of the beam formers with common weights under the factorizations in (13). Substitution of (13) into (30) leads to

$$
\Gamma_{s} G c=\Gamma_{d} H c .
$$

Owing to the uniqueness of pattern factorization, we must have $\boldsymbol{\Gamma}_{s} \boldsymbol{G}=\boldsymbol{\Gamma}_{d} \boldsymbol{H}$, or

$$
\left[\begin{array}{c}
j \boldsymbol{G}(1: J,:) \\
-j \boldsymbol{G}(M-J+1: M,:)
\end{array}\right]=\left[\begin{array}{c}
\boldsymbol{H}(1: J,:) \\
\boldsymbol{H}(M-J+1: M,:)
\end{array}\right]
$$

Note that (33) indicates that the imposition of "common weights" does not affect the "common pattern factor" $c(u)$. Letting $J<L$, as assumed, we now expand on the conditions which $\boldsymbol{g}$ and $\boldsymbol{h}$ must satisfy according to (33).

Case 1) $J \geq N$ : Substitution of (15) into (33) leads to

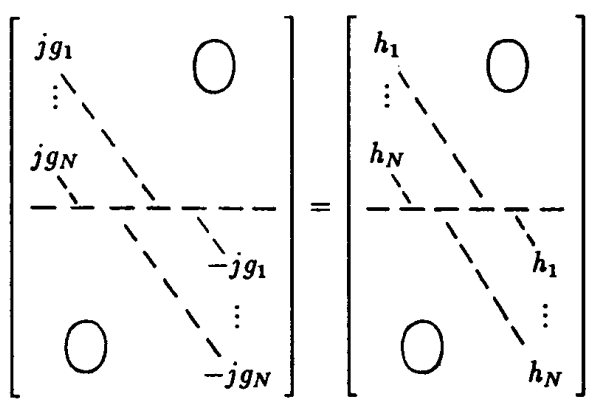

Comparing both sides of (34) leads to $g=h=o_{N}$. We thus conclude that the maximum number of common weights equals $2 J=2(N-1)$.

Case 2) $N+1 \leq 2 J \leq 2(N-1)$ : In this case, we have

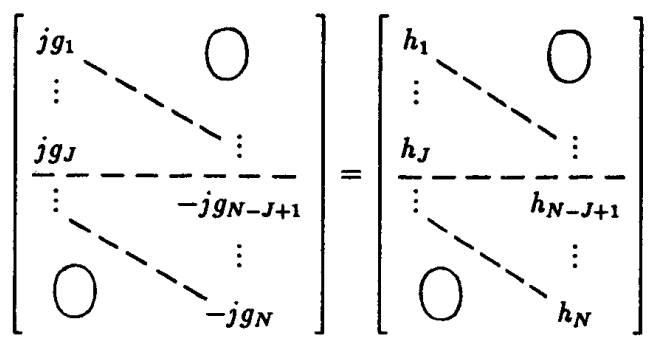

Comparing both sides of (35) leads to

$$
\begin{gathered}
g_{i}=h_{i}=0 \quad i=N-J+1, \cdots, J \\
j g_{i}=h_{i} \quad i=1, \cdots, N-J \\
-j g_{i}=h_{i} \quad i=J+1, \cdots, N .
\end{gathered}
$$

In order to satisfy weights sharing, some of the components of $\boldsymbol{g}$ and $\boldsymbol{h}$ must be zero. This represents a reduction in degree of freedom for synthesizing the patterns.

Now substituting (36) into (15) yields the following expressions for $s$ and $\boldsymbol{d}$ :

$$
\begin{aligned}
s=\overline{\boldsymbol{C}}_{s} \overline{\boldsymbol{h}} & =\boldsymbol{G} \boldsymbol{c} \\
d=\overline{\boldsymbol{C}}_{d} \overline{\boldsymbol{h}} & =\boldsymbol{H} \boldsymbol{c}
\end{aligned}
$$

where

$$
\begin{gathered}
\overline{\boldsymbol{C}}_{s}=[-j \boldsymbol{C}(:, 1: N-J) \mid j \boldsymbol{C}(:, J+1: N)] \\
\overline{\boldsymbol{C}}_{d}=[\boldsymbol{C}(:, 1: N-J) \mid \boldsymbol{C}(:, J+1: N)]
\end{gathered}
$$


and

$$
\overline{\boldsymbol{h}}=\left[\begin{array}{l}
\boldsymbol{h}(1: N-J) \\
\boldsymbol{h}(J+1: N)
\end{array}\right]
$$

It is interesting to see that $\boldsymbol{s}$ and $\boldsymbol{d}$ are now related through $\boldsymbol{c}$ and $\overline{\boldsymbol{h}}$ only. Application of LS-fit procedure in accordance with (37) leads to the two-stage problem described by

$$
\begin{gathered}
\min _{\overline{\boldsymbol{h}}}\left\|\boldsymbol{B} \overline{\boldsymbol{h}}-\boldsymbol{w}_{o}\right\|^{2} \\
\text { subject to: } \quad \boldsymbol{h}^{H} \boldsymbol{a}_{N}(0)=\overline{\boldsymbol{h}}^{H} \mathbf{1}_{2(N-J)}=0,
\end{gathered}
$$

where

$$
\boldsymbol{B}=\left[\begin{array}{l}
\overline{\boldsymbol{C}}_{s} \\
\overline{\boldsymbol{C}}_{d}
\end{array}\right]
$$

and (19), with $\boldsymbol{G}$ and $\boldsymbol{H}$ related according to (36). Note that the subproblem (40) is significantly different in form from that described in (17). The procedure is alternately executed between

$$
\begin{aligned}
\overline{\boldsymbol{h}} & =\left(\boldsymbol{B}^{H} \boldsymbol{B}\right)^{-1} \\
& \cdot\left\{\boldsymbol{I}_{2(N-J)}-\frac{\mathbf{1}_{2(N-J)} \mathbf{1}_{2(N-J)}^{T}\left(B^{H} \boldsymbol{B}\right)^{-1}}{\mathbf{1}_{2(N-J)}^{T}\left(B^{H} B\right)^{-1} \mathbf{1}_{2(N-J)}}\right\} \boldsymbol{B}^{H} \boldsymbol{w}_{o}
\end{aligned}
$$

and (22) until the solutions converge. Following a derivation similar to that in the Appendix, we can show that if $\boldsymbol{s}_{o}, \boldsymbol{d}_{o}$, and the initial guess $c$ are all CS, then the final solutions for $s$ and $d$ will be CS as well.

Case 3) $2 J \leq N$ : Observing again (35) leads to

$$
\begin{gathered}
j g_{i}=h_{i} \quad i=1, \cdots, J \\
-j g_{i}=h_{i} \quad i=N-J+1, \cdots, N
\end{gathered}
$$

In this case $g_{i}$ and $h_{i}, i=J+1, \cdots, N-J$, are allowed to vary freely. This indicates that some degree of freedom is obtained for synthesizing the patterns by reducing the number of common weights. Application of the LS-fit procedure in accordance with (43) is more involved than case 2 . We first check that $s$ and $\boldsymbol{d}$ have the following structures:

$$
\begin{array}{r}
\boldsymbol{s}=\overline{\boldsymbol{C}}_{s} \overline{\boldsymbol{h}}+\tilde{\boldsymbol{C}} \tilde{\boldsymbol{g}}=\boldsymbol{G} \boldsymbol{c} \\
\boldsymbol{d}=\overline{\boldsymbol{C}}_{d} \overline{\boldsymbol{h}}+\tilde{\boldsymbol{C}} \tilde{\boldsymbol{h}}=\boldsymbol{H c}
\end{array}
$$

where in this case

$$
\begin{gathered}
\overline{\boldsymbol{C}}_{s}=[-j \boldsymbol{C}(:, 1: J) \mid j \boldsymbol{C}(:, N-J+1: N)] \\
\overline{\boldsymbol{C}}_{\boldsymbol{d}}=[\boldsymbol{C}(:, 1: J) \mid \boldsymbol{C}(:, N-J+1: N)] \\
\tilde{\boldsymbol{C}}=\boldsymbol{C}(:, J+1: N-J) \\
\overline{\boldsymbol{h}}=\left[\begin{array}{c}
\boldsymbol{h}(1: J) \\
\boldsymbol{h}(N-J+1: N)
\end{array}\right] \\
\tilde{\boldsymbol{g}}=\boldsymbol{g}(J+1: N-J) \\
\tilde{\boldsymbol{h}}=\boldsymbol{h}(J+1: N-J) .
\end{gathered}
$$

Application of LS-fit procedure now leads to the two-stage problem described by

$$
\min _{\overline{\boldsymbol{h}}, \tilde{\boldsymbol{g}}, \tilde{\boldsymbol{h}}}\left\|\overline{\boldsymbol{C}}_{s} \overline{\boldsymbol{h}}+\tilde{\boldsymbol{C}} \tilde{\boldsymbol{g}}-\boldsymbol{s}_{o}\right\|^{2}+\left\|\overline{\boldsymbol{C}}_{d} \overline{\boldsymbol{h}}+\tilde{\boldsymbol{C}} \tilde{\boldsymbol{h}}-\boldsymbol{d}_{o}\right\|^{2}
$$

subject to: $\quad \boldsymbol{h}^{H} \boldsymbol{a}_{N}(0)=\overline{\boldsymbol{h}}^{H} \mathbf{1}_{2 J}+\tilde{\boldsymbol{h}}^{H} \mathbf{1}_{N-2 J}=0$

and (19), with $\boldsymbol{G}$ and $\boldsymbol{H}$ related according to (43). Note that in the first term of (49), $\overline{\boldsymbol{h}}$ and $\tilde{\boldsymbol{g}}$ are separable parameters such that we may substitute the LS solutions for $\tilde{\boldsymbol{g}}$ :

$$
\tilde{\boldsymbol{g}}=\tilde{\boldsymbol{g}}_{L S}=\left(\tilde{\boldsymbol{C}}^{H} \tilde{\boldsymbol{C}}\right)^{-1} \tilde{\boldsymbol{C}}^{H}\left(\boldsymbol{s}_{o}-\overline{\boldsymbol{C}}_{\boldsymbol{s}} \overline{\boldsymbol{h}}\right)
$$

to get

$$
\begin{aligned}
& \min _{\overline{\boldsymbol{h}}, \tilde{\boldsymbol{h}}}\left\|\boldsymbol{P}_{c}^{\perp} \overline{\boldsymbol{C}}_{s} \overline{\boldsymbol{h}}-\boldsymbol{P}_{c}^{\perp} \boldsymbol{s}_{o}\right\|^{2}+\left\|\tilde{\boldsymbol{C}}_{d} \overline{\boldsymbol{h}}+\tilde{\boldsymbol{C}} \tilde{\boldsymbol{h}}-\boldsymbol{d}_{o}\right\|^{2} \\
& \quad \equiv \min _{\boldsymbol{h}}\left\|\boldsymbol{A} \boldsymbol{h}-\boldsymbol{v}_{o}\right\|^{2} \\
& \text { subject to: } \quad \boldsymbol{h}^{H} \boldsymbol{a}_{N}(0)=\boldsymbol{h}^{H} \mathbf{1}_{N}=0
\end{aligned}
$$

where $\boldsymbol{P}_{\mathrm{c}}^{\perp}, \boldsymbol{A}$, and $\boldsymbol{v}_{o}$ are defined at the bottom of the page. With $\boldsymbol{h}$ determined by

$$
\boldsymbol{h}=\left(\boldsymbol{A}^{H} \boldsymbol{A}\right)^{-1}\left\{\boldsymbol{I}_{N}-\frac{\mathbf{1}_{N} \mathbf{1}_{N}^{T}\left(\boldsymbol{A}^{H} \boldsymbol{A}\right)^{-1}}{\mathbf{1}_{N}^{T}\left(\boldsymbol{A}^{H} \boldsymbol{A}\right)^{-1} \mathbf{1}_{N}}\right\} \boldsymbol{A}^{H} \boldsymbol{v}_{o}
$$

$g$ can be constructed by $\bar{h}$ and $\tilde{g}$ via (43), (47), and (48). The

$$
\begin{aligned}
& \boldsymbol{P}_{c}^{\perp}=\boldsymbol{I}_{M}-\tilde{\boldsymbol{C}}\left(\tilde{\boldsymbol{C}}^{H} \tilde{\boldsymbol{C}}\right)^{-1} \tilde{\boldsymbol{C}}^{H} \\
& \boldsymbol{A}=\left[\begin{array}{ccc}
\boldsymbol{P}_{c}^{\perp} \overline{\boldsymbol{C}}_{s}(:, 1: J) & \boldsymbol{O}_{M \times(N-2 J)} & \boldsymbol{P}_{c}^{\perp} \overline{\boldsymbol{C}}_{s}(:, N-J+1: N) \\
\overline{\boldsymbol{C}}_{d}(:, 1: J) & \tilde{\boldsymbol{C}} & \overline{\boldsymbol{C}}_{d}(:, N-J+1: N)
\end{array}\right] \\
& \boldsymbol{v}_{o}=\left[\begin{array}{c}
\boldsymbol{P}_{c}^{\perp} \boldsymbol{s}_{o} \\
\boldsymbol{d}_{o}
\end{array}\right]
\end{aligned}
$$




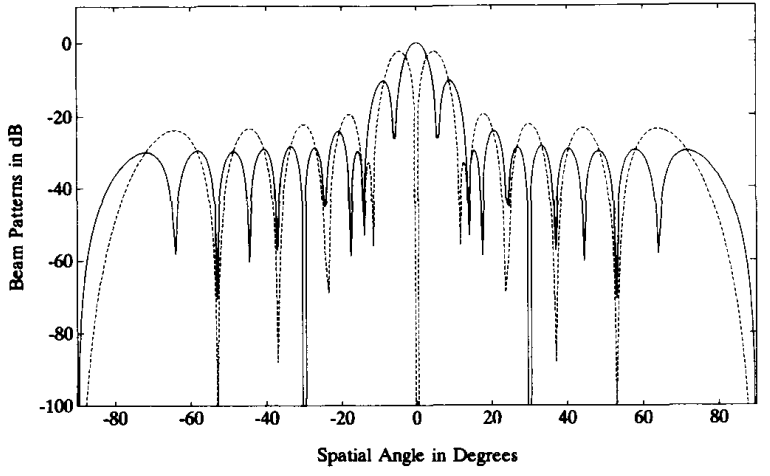

(a)

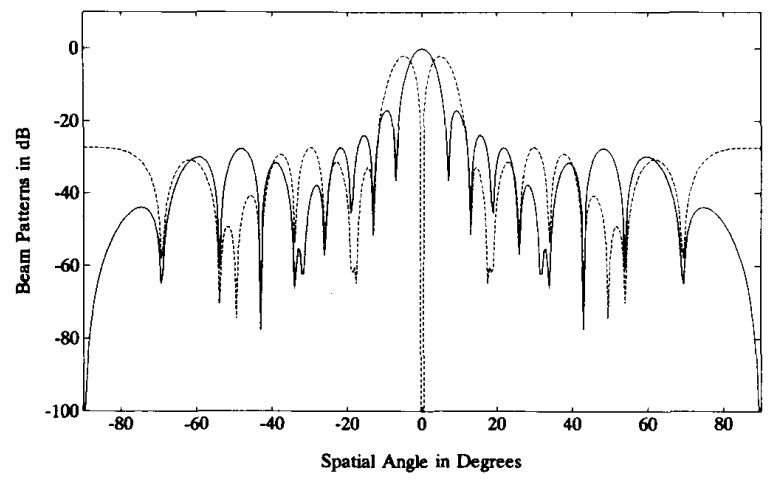

(c)

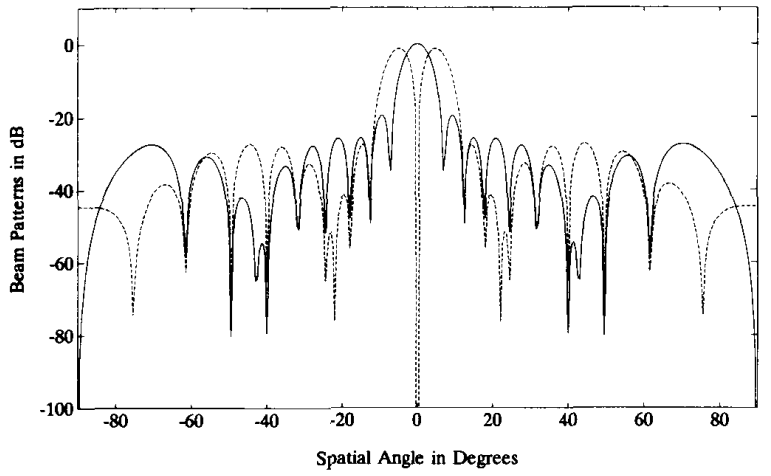

(b)

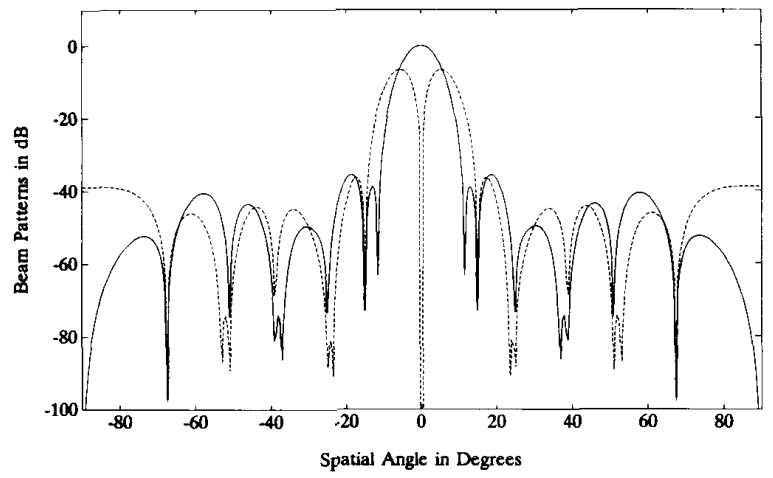

(d)

Fig. 4. Superposition of the subaray-based/LS-fit patterns with common weights synthesized with a 20-element LES array. (a) $N=11, J=10$. (b) $N=6, J=3$. (c) $N=6, J=4$. (d) $N=6, J=5$. Solid line: sum. Dashed line, difference.

procedure is alternately executed between (55) and (22) until the solutions converge.

That the number of common weights is bounded above by $2(N-1)$ represents a design trade-off between system complexity and computational requirement. Observing again the excitation distributions associated with the sum and difference beams, we see that a criterion for choosing $J$ would be $J \approx \frac{M}{4}$. With this selection of $J$, we can reduce the number of independent weights by a factor of $\frac{4}{3}$, and reduce the order of polynomial rooting in bearing estimation by at most a factor of 4 .

\section{B. Synthesis of Patterns in the Presence of Strong Interference}

Incorporation of interference cancellation in accordance (26) still applies since the imposition of common weights does not affect the factor $c$, as stated previously. Hence if adaptive nulling is performed, we simply replace (22) by (28) in the solutions for cases 2 and 3.

\section{Numerical Examples}

The second set of examples investigate the sum and difference patterns synthesized in accordance with the previously developed procedures involving common weights. The array configuration was the same as that used in subsection III-C.
Fig. 4(a) shows the patterns synthesized with $N=11$ and $J=10$ (full common weights). Note that $N=11$ is the minimum value allowable for this case. We see that the approximation between the synthesized and the reference patterns is poor compared with Fig. 2, confirming our earlier comment regarding full common weights implementation. To demonstrate the improvement by using partial common weights, we show in parts (b) through (d) of Fig. 4 the patterns synthesized with $N=6$ and $J=3,4,5$, respectively. Note that $J=3$ corresponds to case 3 , whereas $J=4$, 5 corresponds to case 2 . Comparing the results with those shown in Fig. 4(a), we see that a significant reduction in side-lobe level was achieved with partial common weights implementation. The patterns obtained with $J=5$ exhibit a large deviation from the reference patterns due to the small degree of freedom for $g$ and $\boldsymbol{h}$. Recall that for $N-J=1$, there is only one independent component for $\boldsymbol{g}$ and $\boldsymbol{h}$. For the above four cases, the corresponding weight vectors are listed in Table I to illustrate how the sum and difference beams share the common weights.

To examine the effect of using common weights on adaptive nulling, we repeated the above simulations, with three common nulls placed at $-40^{\circ}, 30^{\circ}$, and $50^{\circ}$. Parts (a) and (b) of Fig. 5 show the resulting patterns synthesized with full common weights and partial common weights with $J=4$, respectively. 
TABLE I

Sum AND DifFERENCE WeIGHT Vectors CoRRESPONDING to THE PatTerns SYNTHESIZED WITH COMMON Weights (Because OF SyMMETRY, ONLY HALF OF THE WEIGHTS ARE LISTED)

\begin{tabular}{|c|cc|cc|cc|cc|}
\hline \multirow{2}{*}{$\begin{array}{c}\text { Array } \\
\text { elemen }\end{array}$} & \multicolumn{7}{|c|}{ Corresponding Figure } \\
\cline { 2 - 9 } index & Fig. 4(a) $(J=10)$ & Fig. 4 (b) $(J=3)$ & Fig.4(c) $(J=4)$ & Fig. 4(d) $(J=5)$ \\
\cline { 2 - 9 } & $\Sigma$ & $\Delta$ & $\Sigma$ & $\Delta$ & $\Sigma$ & $\Delta$ & $\Sigma$ & $\Delta$ \\
\hline 1 & 1.5229 & $j 1.5229$ & 1.0111 & $j 1.0111$ & 1.0185 & $j 1.0185$ & 0.1821 & $j 0.1821$ \\
2 & 1.8671 & $j 1.8671$ & 1.3472 & $j 1.3472$ & 1.3874 & $j 1.3874$ & 0.3511 & $j 0.3511$ \\
3 & 2.3580 & $j 2.3580$ & 1.9505 & $j 1.9505$ & 1.9903 & $j 1.9903$ & 0.8552 & $j 0.8552$ \\
4 & 2.7184 & $j 2.7184$ & 1.7115 & $j 3.2339$ & 2.5192 & $j 2.5192$ & 1.4160 & $j 1.4160$ \\
5 & 2.9134 & $j 2.9134$ & 2.2749 & $j 3.5294$ & 2.2088 & $j 3.6303$ & 1.9850 & $j 1.9850$ \\
6 & 2.9134 & $j 2.9134$ & 2.5068 & $j 3.7054$ & 2.6657 & $j 3.5573$ & 2.6461 & $j 2.2819$ \\
7 & 2.7184 & $j 2.7184$ & 2.6754 & $j 3.5350$ & 2.7616 & $j 3.3869$ & 3.1432 & $j 2.4409$ \\
8 & 2.3580 & $j 2.3580$ & 2.7797 & $j 2.8833$ & 2.8105 & $j 2.7824$ & 3.7591 & $j 2.0487$ \\
9 & 1.8671 & $j 1.8671$ & 2.8356 & $j 1.9242$ & 2.8198 & $j 1.8367$ & 4.2080 & $j 1.3761$ \\
10 & 1.5229 & $j 1.5229$ & 2.8585 & $j 0.6502$ & 2.8187 & $j 0.6364$ & 4.4490 & $j 0.4790$ \\
\hline
\end{tabular}

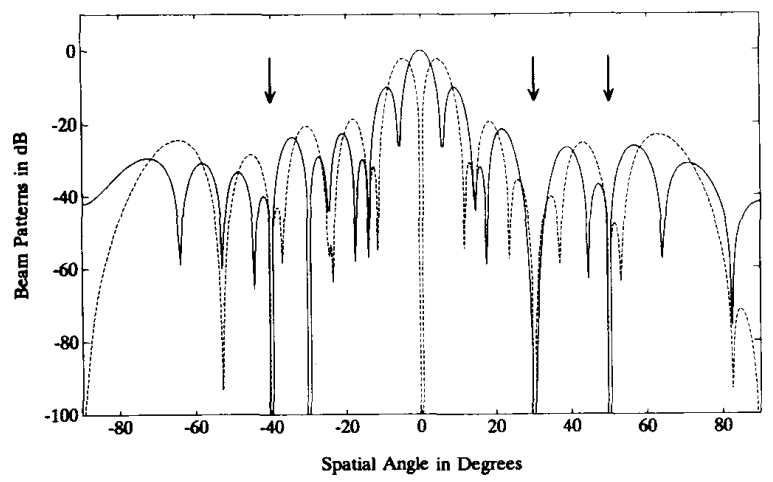

(a)

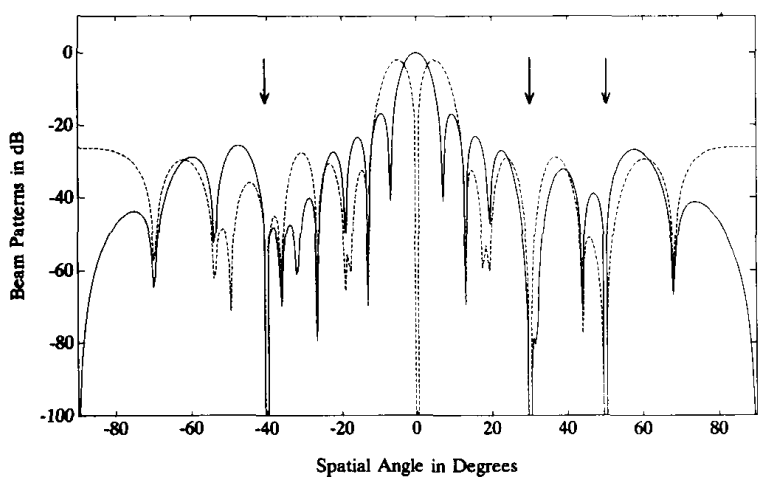

(b)

Fig. 5. Superposition of the subaray-based/LS-fit interference cancellation patterns with common weights synthesized with a 20-element LES array. (a) $N=11, J=10$. (b) $N=6, J=4$. Solid line: sum. Dashed line difference.

The corresponding weights are listed in Table II. The three nulls were successfully formed as dictated, without affecting the synthesized patterns much. We note from Table II that the weight vectors exhibit nonzero real and imaginary parts, as opposed to the case without interference cancellation. This is due to the loss of symmetry of the null distributions in
TABLE II

SuM AND DifFERENCE WeIGHT VeCtORS CORRESPONDING TO THE INTERFERENCE CANCELLATION PATTERNS SYNTHESIZED WITH COMMON Weights (Because of Symmetry, ONLy Half of the Weights ARE Listed)

\begin{tabular}{|c|cr|rr|}
\hline \multirow{2}{*}{$\begin{array}{c}\text { Array } \\
\text { element }\end{array}$} & \multicolumn{4}{|c|}{ Corresponding Figure } \\
\cline { 2 - 5 } index & \multicolumn{2}{|c|}{ Fig. 5(a) $(J=10)$} & \multicolumn{2}{c|}{ Fig. 5(b) $(J=4)$} \\
\cline { 2 - 5 } & $\Sigma$ & $\Delta$ & $\Sigma$ & $\Delta$ \\
\hline 1 & $1.3235+j 0.1800$ & $-0.1800+j 1.3235$ & $0.9055+j 0.0396$ & $-0.0396+j 0.9055$ \\
2 & $1.7943-j 0.0851$ & $0.0851+j 1.7943$ & $1.2826-j 0.0437$ & $0.0437+j 1.2826$ \\
3 & $2.5370-j 0.1822$ & $0.1822+j 2.5370$ & $2.0980-j 0.0003$ & $0.0003+j 2.0980$ \\
4 & $2.7089+j 0.1396$ & $-0.1396+j 2.7089$ & $2.4932-j 0.0238$ & $0.0238+j 2.4932$ \\
5 & $2.8618+j 0.1468$ & $-0.1468+j 2.8618$ & $2.2471+j 0.0273$ & $-0.0822+j 3.5021$ \\
6 & $2.8618-j 0.1468$ & $0.1468+j 2.8618$ & $2.6803+j 0.0906$ & $0.0112+j 3.5169$ \\
7 & $2.7089-j 0.1396$ & $0.1396+j 2.7089$ & $2.6258-j 0.1593$ & $0.1428+j 3.5483$ \\
8 & $2.5370+j 0.1822$ & $-0.1822+j 2.5370$ & $2.9059+j 0.0852$ & $-0.0643+j 2.8050$ \\
9 & $1.7943+j 0.0851$ & $-0.0851+j 1.7943$ & $2.8366+j 0.0533$ & $-0.1624+j 1.7646$ \\
10 & $1.3235-j 0.1800$ & $0.1800+j 1.3235$ & $2.8193-j 0.1476$ & $0.1266+j 0.6220$ \\
\hline
\end{tabular}

the sum and difference patterns. Amplitude-only technique [3] may be employed to recover the symmetry. However, the price paid is that the maximum number of jammers which can be eliminated is halved.

Again, for all of the above examples, the initial guess of $c$ was chosen as $c=1 \mathrm{~K}$. The algorithm was terminated if $\left\|c^{k+1}-c^{k}\right\|<0.01\left\|c^{k}\right\|$. For this set of examples, the algorithm converged, on an average, within five iterations.

\section{CONCLUSION}

A class of design criteria has been proposed for the synthesis of low-side-lobe sum and difference patterns for monopulse LES arrays. The idea of subarray beam forming was proposed as a means of reducing the computational complexity in target bearing estimation. A LS-fit procedure was developed for constructing the optimum sum and difference patterns possessing a common factor which are closest to a set of reference patterns in the LS sense. Adaptive nulling was incorporated to enhance the estimation performance under strong out-ofband interference. To further ease the system complexity, we imposed the constraint that the sum and difference channels share a subset of the beam-forming weights. The relationship between the maximum number of common weights allowable and the number of subarrays used is discussed in detail. It is found that the appropriate configuration is that one fourth the weights from both ends of the array aperture are common. In this case, the number of independent weights can be reduced by a factor of $4 / 3$, and the order of polynomial rooting required for bearing estimation can be reduced by at most a factor of 4 .

\section{APPENDIX}

\section{CS PROPERTY OF LS-FIT SUM AND} DIFFERENCE WEIGHT VECTORS

We first consider the following lemma.

Lemma: If $\boldsymbol{c}$ is $\mathrm{CS}$, i.e., $\tilde{\boldsymbol{I}}_{K} \boldsymbol{c}=\boldsymbol{c}^{*}$, then $\boldsymbol{C}$ satisfies $\tilde{I}_{M} C \tilde{I}_{N}=C^{*}$, where $\tilde{I}_{K}$ and $\tilde{I}_{N}$ are given by (24), with $M=K$ and $N$, respectively. 
Proof:

$$
\begin{aligned}
& \tilde{\mathbf{I}}_{M} \mathrm{C} \tilde{\mathbf{I}}_{N}=\tilde{\mathbf{I}}_{M}\left[\begin{array}{cc}
c_{1} & 0 \\
\vdots & c_{1} \\
c_{K} & \vdots \\
0 & c_{K}
\end{array}\right] \tilde{\mathbf{I}}_{N}=\left[\begin{array}{cc}
c_{K} & 0 \\
\vdots & c_{K} \\
c_{1} & \vdots \\
0 & c_{1}
\end{array}\right] \\
& =\left[\begin{array}{cc}
c_{1}^{*} & 0 \\
\vdots & c_{1}^{*} \\
c_{K}^{*} & \vdots \\
O^{-} & c_{K}^{*}
\end{array}\right]=\mathrm{C}^{*}
\end{aligned}
$$

If $s_{o}$ is also CS, i.e., $\tilde{I}_{M} s_{o}=s_{o}^{*}$, then we have

$$
\begin{aligned}
\tilde{\boldsymbol{I}}_{N} \boldsymbol{g} & =\tilde{\boldsymbol{I}}_{N}\left(\boldsymbol{C}^{H} \boldsymbol{C}\right)^{-1} \boldsymbol{C}^{H} \boldsymbol{s}_{o} \\
& =\tilde{\boldsymbol{I}}_{N}\left(\boldsymbol{C}^{H} \boldsymbol{C}\right)^{-1} \tilde{\boldsymbol{I}}_{N} \tilde{\boldsymbol{I}}_{N} \boldsymbol{C}^{H} \tilde{\boldsymbol{I}}_{M} \tilde{\boldsymbol{I}}_{M} \boldsymbol{s}_{o} \\
& =\left(\tilde{\boldsymbol{I}}_{N} \boldsymbol{C}^{H} \boldsymbol{C} \tilde{\boldsymbol{I}}_{N}\right)^{-1} \tilde{\boldsymbol{I}}_{N} \boldsymbol{C}^{H} \tilde{\boldsymbol{I}}_{M} \tilde{\boldsymbol{I}}_{M} \boldsymbol{s}_{o} \\
& =\left(\tilde{\boldsymbol{I}}_{N} \boldsymbol{C}^{H} \tilde{\boldsymbol{I}}_{M} \tilde{\boldsymbol{I}}_{M} \boldsymbol{C} \tilde{\boldsymbol{I}}_{N}\right)^{-1} \tilde{\boldsymbol{I}}_{N} \boldsymbol{C}^{H} \tilde{\boldsymbol{I}}_{M} \tilde{\boldsymbol{I}}_{M} \boldsymbol{s}_{o} \\
& =\left(\boldsymbol{C}^{T} \boldsymbol{C}^{*}\right)^{-1} \boldsymbol{C}^{T} \boldsymbol{s}_{o}^{*} \\
& =\left[\left(\boldsymbol{C}^{H} \boldsymbol{C}\right)^{-1} \boldsymbol{C}^{H} \boldsymbol{s}_{o}\right]^{*} \\
& =\boldsymbol{g}^{*}
\end{aligned}
$$

where we have used $\tilde{I}_{M} \tilde{I}_{M}=I_{M}$ and $\tilde{I}_{N} \tilde{I}_{N}=I_{N}$. Hence $g$ is also CS.

The proof for the CS property of $h$ and $c$ is similar.

\section{REFERENCES}

[1] R. S. Elliott, "Design of line source antennas for difference patterns with sidelobes of individually arbitrary heights," IEEE Trans. Antennas Propagat. vol. 24, pp. 310-316, May 1976.

[2] R. L. Haupt, "Simultaneous nulling in the sum and difference patterns of a monopulse antenna," IEEE Trans. Antennas Propagat., vol. 32, pp. 486-493, May 1984

[3] T. B. Vu, "Simultaneous nulling in sum and difference pattern by amplitude control," IEEE Trans. Antennas Propagat., vol. 34, pp. 214-218, Feb. 1986.

[4] D. A. Mcnamara "Synthesis of sub-arrayed monopulse linear arrays through matching of independently optimun sum and difference excitations," Proc. Inst. Elec. Eng., vol. 135, pt. H, no. 5, pp. 293-296, Oct. 1988.

[5] R. L. Haupt, "Adaptive nulling in monopulse antennas," IEEE Trans. Antennas Propagat., vol. 36, pp. 202-208, Feb. 1988.
[6] C. L. Dolph, "A current distribution for broadside arrays which optimizes the relationship between beamwidth and side lobe level," Proc. IRE, vol. 34, pp. 335-348, 1946

[7] E. T. Bayliss, "Design of monopulse antenna difference patterns with low side lobes," Bell Syst. Tech. J., vol. 47, pp. 632-640, 1968.

[8] S. M. Sherman, Monopulse Principle and Techniques. Norwood, MA Artech House, 1985.

[9] M. I. Skolnik, Introduction to Radar Systems. New York: McGrawHill, 1962.

[10] R. C. Davis, L. E. Brennan, and L. S. Reed "Angle estimation with adaptive arrays in external noise field," IEEE Trans. Aerosp. Electron. Syst., vol. 12, pp. 179-186, Nov. 1976.

[11] W. D. White, "Low angle radar tracking in the presence of multipath," IEEE Trans. Aerosp. Electron. Syst., vol. 10, pp. 835-853, Nov. 1974.

[12] B. D. Steinberg, Principle of Aperture and Array System Design. New York, NY: John Wiley, 1976

[13] A. J. Weiss and B. Friedlander, "Array shape calibration using sources in unknown locations-A maximum likelihood approach," IEEE Trans. Acoust., Speech, Signal Process. vol. 37, pp. 1958-1966, Dec. 1989.

[14] E. Brookner and J. Howells, "Adaptive-adpative array processing," Proc IEEE, vol. 74, pp. 602-604, Apr., 1986.

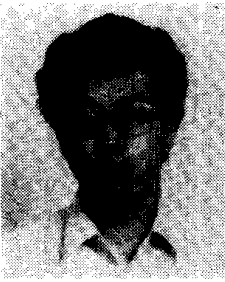

Ta-Sung Lee (S'88-M'89) was born in Taipei, Taiwan, Republic of China, on October 20, 1960. He received the B.S. degree from National Taiwan University in 1983, the M.S. degree from the University of Wisconsin at Madison in 1987, and the Ph.D. degree from Purdue University in 1989, all in electrical engineering.

From 1987 to 1989 he was a David Ross Graduate Research Fellow at Purdue University. In the spring of 1990 he joined the faculty of National Chiao Tung University, where he is currently an associate professor in the Department of Communication Engineering. His present research interests include sensor array signal processing, adaptive antennas for mobile communications, underwater acoustic signal processing, and blind equalization.

Dr. Lee is a member of Phi Tau Phi.

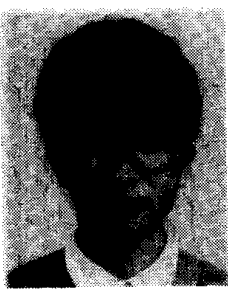

Te-Kung Tseng was born in Keelung, Taiwan, Republic of China, on October 1, 1967. He received the B.S. degree in electrical engineering from the National Taiwan Institute of Technology in 1990 and the M.S. degree in communication engineering from National Chiao Tung University in 1992.

From 1990 to 1992 he was a research assistant at Chiao Tung University. In August 1992 he joined the TECOM Corporation in Science-Based Industrial Park, Hsinchu, Taiwan, where he is currently an $R \& D$ engineer. 